\title{
La formation et le développement du système éducatif public mexicain
}

Yves Robin

\section{OpenEdition}

1 Journals

Édition électronique

URL : https://journals.openedition.org/ries/786

DOI : $10.4000 /$ ries.786

ISSN : 2261-4265

Éditeur

France Education international

\section{Édition imprimée}

Date de publication : 1 décembre 2009

Pagination : 143-148

ISBN : 978-2-8542-0577-0

ISSN : $1254-4590$

Référence électronique

Yves Robin, «La formation et le développement du système éducatif public mexicain », Revue internationale d'éducation de Sèvres [En ligne], 52 I décembre 2009, mis en ligne le 01 décembre 2011, consulté le 27 avril 2022. URL : http://journals.openedition.org/ries/786 ; DOI : https://doi.org/ 10.4000/ries.786

Ce document a été généré automatiquement le 27 avril 2022

(c) Tous droits réservés 


\title{
La formation et le développement du système éducatif public mexicain
}

\author{
Yves Robin
}

Durant la colonisation espagnole (1521-1821), l'Église domine la société mexicaine du point de vue économique - c'est l'une des plus puissantes d'Amérique latine - et détient le contrôle quasi exclusif de l'institution scolaire par l'intermédiaire de différents ordres religieux dont les Franciscains, les Dominicains et surtout les Jésuites, arrivés en Nouvelle Espagne au cours du XVIe siècle. Ce monopole est remis en cause au moment de l'indépendance (1821) et, en 1823, se crée une direction nationale de l'instruction afin d'établir, conserver et améliorer l'instruction publique dans toute la nation: l'éducation est déclarée publique, gratuite et identique pour tous. Ainsi, le nouvel État se voit-il conférer légalement des prérogatives en matière d'éducation. Toutefois, compte tenu de la situation politique, économique et sociale chaotique, il ne pourra jamais les exercer. Selon Fernando Solana, historien mexicain, l'éducation publique mexicaine naît avec le libéralisme, en 1833, suite aux lois promulguées par Gomez Farias, qui créent une direction générale de l'instruction publique et définissent clairement, pour la première fois, les compétences de l'État dans le domaine éducatif. Le projet d'éducation publique, gratuite, laïque sera renforcé avec le triomphe de la Réforme $^{1}$ mais il faudra attendre la fin de l'intervention française (1862-1867) pour qu'il connaisse un début d'application.

\section{Victoire des libéraux sur l'Empire et apport du positivisme comtien}

2 La victoire du libéral Benito Juárez sur l'Empire de Maximilien restaure la république. Elle renforce surtout le sentiment patriotique et contribue à forger l'unité nationale : en ce sens, elle peut être considérée comme une « seconde indépendance ». Les libéraux sont alors confrontés à la question de la mise en place de nouvelles institutions : il s'agit de concrétiser un modèle social dont l'École sera la pierre angulaire. En effet, ils ont toujours proclamé leur foi dans l'institution scolaire et ils s'attachent dès 1867 à créer 
de nombreuses écoles au moyen de la publication par Gabino Barreda, ministre de l'éducation de Benito Juárez et adepte du positivisme comtien, d'une loi organisant l'instruction publique. Outre la création d'établissements scolaires (dans ce domaine, le pays a un retard considérable), cette loi propose d'unifier l'enseignement élémentaire en affirmant son caractère obligatoire et gratuit. Deux ans plus tard, une autre loi vient compléter la première en supprimant l'enseignement religieux à l'école et en proclamant, pour la première fois, la laïcité de l'enseignement public. Dans cette dernière partie du XIX ${ }^{e}$ siècle, la philosophie positiviste incarne la modernité et s'oppose à la conception religieuse de l'éducation. Elle devient une idéologie directrice dans toutes les politiques éducatives, processus qui se prolongera durant le Porfiriat ${ }^{2}$ et jusqu'à la révolution (1910-1920). En cette fin de XIX ${ }^{e}$ siècle, les dirigeants politiques affirment leur volonté de renforcer l'unité mexicaine et considèrent que la question de l'éducation est essentielle dans la perspective de l'élaboration d'une conscience nationale. Pour illustrer cette détermination, le premier congrès national pour l'instruction publique est inauguré en 1899, à Mexico, sous la présidence de Justo Sierra, ministre de l'instruction publique et grande figure du positivisme: à cette occasion, le caractère laïc, gratuit et obligatoire de l'enseignement élémentaire est réaffirmé. Mais ce n'est qu'en 1917, avec la constitution «révolutionnaire » de Querétaro, que sera établi de manière définitive le principe de la prééminence de l'État en matière éducative et d'un enseignement scolaire laïc et gratuit. En 1921, José Vasconcelos (1882-1959) personnage clé de l'éducation au Mexique, crée le ministère de l'éducation publique et des beaux-arts qui existe toujours sous cette forme. Il fait de la question éducative une véritable croisade nationale. Ardent défenseur d'une éducation laïque et populaire, il met en place l'éducation indigène, l'éducation rurale et technique. Il crée des réseaux de bibliothèques, des missions culturelles, les écoles normales pour la formation des enseignants et les Casas de Pueblo, centres d'éducation élémentaire et d'activités socioculturelles. C'est grâce à son engagement et avec l'appui des dirigeants issus de la révolution que le système éducatif public mexicain va se développer d'une manière significative.

\section{Des manuels scolaires publics gratuits, uniques et obligatoires}

Néanmoins, il faudra attendre les années cinquante et une certaine croissance économique liée à une politique nationaliste pour que le président Adolfo Lopez Mateos (1958-1964³) installe d'une manière définitive et durable le système éducatif public. Pour cela, il impose un effort budgétaire sans précédent dans l'histoire de la nation. Cette politique volontariste se concrétise par une importante réforme connue sous le nom de «Plan d'amélioration de l'enseignement primaire » appelé aussi «Plan de onze ans ». L'objectif principal est de garantir à tous les enfants mexicains une éducation primaire obligatoire et gratuite, conformément à l'article 3 de la constitution de 1946. En 1958 en effet, on estime qu'environ $40 \%$ des enfants d'âge scolaire - de 6 à 14 ans - ne sont pas scolarisés. Compte tenu de la croissance démographique, le gouvernement annonce qu'il devient indispensable de résoudre ce problème qui met en péril le développement économique du pays. Pour mener à bien ce projet, il est donc nécessaire de construire des écoles, de former des maîtres et d'élaborer puis de distribuer gratuitement des manuels scolaires appelés libros de texto gratuitos (livres 
de textes gratuits), supports indispensables de cette politique ${ }^{4}$. Afin de mieux comprendre l'enjeu de cette politique et l'impact qu'elle a pu avoir sur la population mexicaine, il convient d'ajouter que la distribution de ces livres scolaires sera la première opportunité pour des millions de Mexicains, qui manquent de ressources économiques et/ou vivent dans des contrées isolées, conséquence de la géographie très accidentée du Mexique, de se confronter à un support culturel écrit. En 1960, le ministère de l'éducation nationale les déclare obligatoires et uniques ${ }^{5}$. De cette manière, l'élaboration, la fabrication et la distribution des manuels scolaires destinés à l'école primaire deviennent un monopole d'État ${ }^{6}$. La création de ces manuels scolaires, considérée comme une véritable "conquête sociale" peut être perçue comme l'aboutissement d'un long processus qui a commencé au lendemain de l'indépendance, lorsque le nouvel État a voulu briser le monopole de l'Église en matière d'éducation. D'un point de vue pédagogique, ces premiers ouvrages sont très influencés par le modèle didactique français. Comme les manuels français de la première moitié du $\mathrm{XX}^{\mathrm{e}}$ siècle, ils sont élaborés en fonction d'une pédagogie basée sur l'apprentissage collectif et la mémorisation. L'organisation des leçons dans les livres d'histoire représente l'exemple le plus marquant de cette influence: non seulement on y trouve des illustrations extraites des manuels français (par exemple la prise de la Bastille, Napoléon franchissant le pont d'Arcole ou des portraits de Voltaire et Montesquieu), mais le découpage des leçons reprend pratiquement à l'identique celui des manuels français. Ce phénomène s'explique par le grand prestige dont la France jouit au Mexique - et dans toute l'Amérique latine - où elle est encore considérée comme le pays des droits de l'homme dont le rayonnement culturel a éclairé l'ensemble de la planète.

4 En 1972, sous la présidence de Luis Echeverría (1970-1976) intervient une nouvelle réforme au cours de laquelle, après plus de douze années d'existence sans changement, le gouvernement mexicain décide d'actualiser et d'améliorer les manuels scolaires. Cette mutation s'élabore dans un climat de crise internationale de la jeunesse symbolisé par le mouvement étudiant de 1968 qui exige des réformes politiques, sociales et éducatives. Après le massacre de la place des Trois Cultures, le 2 octobre 1968, à Mexico ${ }^{7}$ sous le mandat du président Díaz Ordaz (1964-1970), le gouvernement de Luis Echeverría se trouve dans l'obligation de répondre à la demande de changement et engage une politique dite "d'ouverture démocratique». Dans ce contexte, il opte pour une réforme éducative en rupture avec les précédentes, dont le principal objectif était de consolider l'unité nationale en déclarant offrir des chances de réussite à tous les Mexicains, en particulier aux plus défavorisés. De nouveaux manuels scolaires sont élaborés à partir de 1971 et publiés entre 1972 et 1974. D'un point de vue didactique, il est décidé de répartir les matières en quatre domaines: les sciences naturelles, les sciences sociales, la langue et les mathématiques. Ainsi disparaît la dénomination " histoire ", cette matière étant intégrée dans le champ des "sciences sociales " en compagnie de la géographie et de l'éducation civique. Cette nouvelle organisation vient concrétiser l'idée que l'école, plus que de transmettre des connaissances, doit développer des attitudes d'expérimentation, de réflexion, d'auto-apprentissage, afin de préparer les nouvelles générations à une culture scientifique et technologique qui caractérisera le monde à venir.

Malgré les changements radicaux dans la politique éducative, le modèle français demeure encore très présent. Ainsi, dans les manuels de littérature et d'histoire, la 
culture française bénéficie d'une large représentation, les auteurs affirmant clairement son rôle dans les changements fondamentaux des sociétés, aux XIX ${ }^{\mathrm{e}}$ et $\mathrm{XX}^{\mathrm{e}}$ siècles.

À la même époque, en France, dans le cadre de la rénovation des programmes scolaires, l'histoire est intégrée aux « activités d'éveil » qui comprennent aussi la géographie et l'éducation civique. Comme au Mexique, il s'agit de favoriser l'éveil des apprenants au moyen d'activités pédagogiques motivantes et formatrices basées sur la découverte et la communication.

7 En 1989, le gouvernement du président Carlos Salinas de Gortari (1988-1994) présente un plan de rénovation de l'enseignement connu sous le nom de «Programme pour la modernisation éducative ». Le changement le plus significatif est l'abandon des quatre domaines qui structuraient l'enseignement des disciplines: après vingt ans d'application d'une réforme, l'institution scolaire reconnaît officiellement son échec et rétablit l'enseignement spécifique de l'espagnol, des mathématiques mais aussi des sciences naturelles, de la géographie et de l'histoire. Ces manuels, toujours en vigueur dans les écoles élémentaires mexicaines, constituent un retour à un enseignement plus patriotique marqué par une utilisation pédagogique plus importante de l'iconographie. Une fois encore, cette évolution fait écho à un changement de perspective de l'enseignement en France où, dès les années 1980, on rétablit l'apprentissage spécifique de l'histoire et on assiste à un recentrage sur l'acquisition d'un petit nombre de connaissances claires et précises susceptibles de servir à la compréhension du monde et de la société dans lesquels vivent les élèves. Face à l'échec proclamé des réformes précédentes - éparpillement des apprentissages, perte des repères chronologiques, désintérêt pour la discipline historique -, on observe que de part et d'autre de l'Atlantique, les autorités éducatives sont revenues à une conception plus traditionnelle de l'enseignement.

\section{Le système éducatif actuel}

8 Depuis les années soixante et la création des livres de textes gratuits, la scolarisation a sensiblement progressé au Mexique malgré une pression démographique toujours importante. Toutefois, la principale préoccupation des dirigeants politiques en matière d'éducation reste l'analphabétisme, qui touchait encore $12 \%$ de la population en 1990. En 2000, le ministère de l'éducation publique estimait que seuls 9,6\% des Mexicains de plus de 15 ans pouvaient être considérés comme analphabètes, avec de grandes disparités régionales puisque ce taux variait de $24 \%$ dans l'État du Chiapas à $3 \%$ dans le district fédéral de Mexico. En 2000, le système éducatif scolarisait environ 29 millions d'élèves de l'école maternelle à l'université, dont $85 \%$ dans des établissements publics.

L'enseignement préélémentaire, qui correspond à l'école maternelle en France, comporte trois niveaux et n'est pas obligatoire. L'école élémentaire comporte six niveaux (un de plus qu'en France) alors que le secondaire n'en comporte que trois. Cette organisation peut s'expliquer par le fait que, jusque dans les années quatre-vingtdix, seul l'enseignement primaire était obligatoire. Ces six niveaux permettaient donc de scolariser les enfants jusqu'à l'âge de 14 ans. On peut comparer ce système à celui en vigueur en France dans les années soixante, où certains élèves effectuaient une année supplémentaire dans le cycle primaire appelée " année de fin d'études ».

L'enseignement secondaire s'adresse aux élèves de 12 à 16 ans, il est obligatoire depuis 1993. Il peut être prolongé par un enseignement «moyen supérieur », qui correspond 
au deuxième cycle de l'enseignement secondaire en France - ou lycée - et qui, à l'issue de trois années, débouche sur l'équivalent du baccalauréat. L'obtention de ce diplôme permet d'intégrer l'enseignement supérieur soit à l'université, soit dans des instituts de technologie ou encore dans des écoles supérieures dites «normales » préparant à la profession d'enseignant. Ces études supérieures sont sanctionnées par une licence obtenue en quatre ans. Puis les étudiants ont la possibilité de préparer une maîtrise en deux ans ou un doctorat en trois ans. Ce système est complété par un enseignement pour les adultes de plus de quinze ans qui, pour diverses raisons, n'ont pas suivi ou terminé l'enseignement primaire.

11 Parallèlement au système d'éducation public, il existe un certain nombre d'établissements privés nationaux ou étrangers - dont les écoles françaises de Mexico, Guadalajara et Cuernavaca - où sont scolarisés les enfants issus des classes favorisées de la société mexicaine. Cette désaffection relative pour le système public a deux principales explications : dans un pays où $90 \%$ de la population se déclare catholique, seules les écoles privées sont autorisées à dispenser un enseignement religieux; face aux défis démographiques (en 2004, $32 \%$ de la population avait moins de 14 ans), le système d'éducation public pâtit d'une pénurie de moyens qui le discrédite aux yeux d'une partie de la population.

12 Pour illustrer ce second point, il est intéressant de rappeler une particularité de fonctionnement du système scolaire mexicain : compte tenu du grand nombre d'élèves, les écoles primaires sont utilisées par des groupes d'apprenants et d'enseignants différents le matin et l'après-midi. En effet, une partie des enfants effectue sa « journée » de classe durant la matinée - «el turno matutino » de huit à treize heures environ -, l'autre partie durant l'après-midi - «el turno vespertino » de treize à dixhuit heures environ. Le soir, ces mêmes écoles sont destinées à l'enseignement pour les adultes. Ainsi, et compte tenu du niveau relativement peu élevé de leurs rémunérations, la plupart des enseignants mexicains font une «journée » de classe le matin et une autre l'après-midi, souvent dans des écoles différentes, publiques et/ou privées, et parfois avec des fonctions différentes : par exemple, un instituteur dans une école le matin peut exercer les fonctions de directeur dans une autre école l'après-midi. Ce système permet aux enseignants de doubler leur salaire et donc de vivre un peu plus confortablement. Naturellement, ces conditions de travail ne sont pas sans répercussion sur la manière d'enseigner. Les maîtres saturés d'heures de classe environ une cinquantaine d'heures hebdomadaires - ne disposent pas du temps nécessaire pour se former, s'informer et préparer leur cours. Ils s'en remettent exclusivement aux manuels en ignorant, la plupart du temps, d'autres matériels didactiques existants. Leurs cours, face à des classes dont les effectifs peuvent atteindre une quarantaine d'élèves, sont le plus souvent magistraux et ils doivent se contenter de résumer, de lire ou de faire lire les manuels en demandant aux apprenants de mémoriser les notions les plus importantes. Dans ce contexte sociopolitique où le système éducatif manque de moyens financiers pour se transformer et où les syndicats dépendants du parti qui a été au pouvoir pendant soixante-dix ans ${ }^{8}$ utilisent leur influence pour entraver les réformes, on constate que la situation de l'enseignement public se dégrade : selon les statistiques officielles, parmi les trente-deux États du pays, dix scolarisent à peine $90 \%$ des enfants et le taux d'analphabétisme atteint plus de $20 \%$ de la population dans trois d'entre eux. 


\section{NOTES}

1. La période dite de la Réforme correspond à la seconde moitié du XIX ${ }^{\mathrm{e}}$ siècle, au cours de laquelle les «libéraux » seront principalement au pouvoir et promulgueront la Constitution de 1857.

2. On désigne par « Porfiriat » la période (entre 1876 et 1910) durant laquelle Porfirio Díaz dirigea le Mexique.

3. Au Mexique, depuis la révolution, les présidents sont élus pour une durée de six ans.

4. Jusqu'alors, les manuels scolaires étaient élaborés par des auteurs privés, publiés par différents éditeurs et achetés par les familles dans les librairies.

5. De 1960 à 1964, le gouvernement édite et distribue 107155755 livres et cahiers d'exercices et 494255 livres du maître, qui doivent couvrir la totalité des besoins de toutes les écoles primaires du pays; ils sont distribués avec l'aide de l'armée et par tous les moyens de locomotion adaptés, afin de fournir les écoles situées dans les endroits les plus reculés du pays.

6. On retrouve un tel système dans quelques pays, notamment en ex-URSS, en Chine, au Vietnam, en ÉGYPTE, EN SYRIE ET AU MAROC.

7. Le 2 octobre 1968, quelques jours avant le début des Jeux olympiques, une manifestation d'étudiants tourna au massacre. Officiellement, le bilan fut de 33 morts. Cependant d'autres sources font état de 200 à 300 victimes. Au moment des faits, Echeverria était le ministre de l'intérieur du président Díaz Ordaz.

8. Il s'agit du PRI (Partido Revolucionario Institucional) crée en 1929 qui est resté au pouvoir jusqu'en 2000 .

\section{INDEX}

Mots-clés : école, enseignement, sciences de l'éducation

Palabras claves : escuela, enseñanza, ciencias de la educación

Keywords : school, teaching, educational sciences

Index géographique : Mexique

\section{AUTEUR}

\section{YVES ROBIN}

Yves Robin est docteur en histoire de l'université de Puebla (Mexique) et docteur en civilisation latino-américaine de l'université de Nantes (thèse en cotutelle sur l'image de la France dans les manuels scolaires mexicains, 1960-2000). Il est actuellement professeur des écoles et enseigne à l'Institut de recherche et de formation en français langue étrangère de l'université de Nantes. 